\title{
Private practice on life support in America
}

A ccountable care organizations, bundled or gain-sharing payment plans and all other manner of alternative health care delivery and physician compensation models. Value-based payment modifiers that account for actual cost of practice and quality of care provided. Medicare economic index weights, geographic practice cost indices and multiple procedure payment reduction policies.

Does anyone, let alone the average physician, comprehend the American health care system anymore?

Not a chance, says Alabama general internist Dr. Richard Esham. "Every physician who's practising can't comprehend all of this, there's no way. It's so vast. It's so diverse. There's so many different, differing ideas about how the American health care system ought to work and how it ought to be paid for. And the average doc can't get his arms around it."

It's leading to what Esham and others say is the most substantial transformation of the American health care system in history - an exodus from private practice to a hospital-employee model of practice or some other manner of team, large group, networked or integrated practice in which "employed physicians" can just see patients and let someone else deal with the complexities of government regulation or the nuances of payment methodologies such as "withholds and risk pools," or "shared savings."

Or, as several delegates told the 2012 American Medical Association (AMA) Annual Meeting in Chicago, Illinois, "the private practitioner is becoming a dinosaur."

The administrative headaches have become so vast that many doctors just want to wash their hands of the "business" side of American medicine and just "do the health care," says Esham, once a "country doctor" and hospital administrator who now is medical director for Computer Programs and Systems Incorporated (CPSI), a soft-

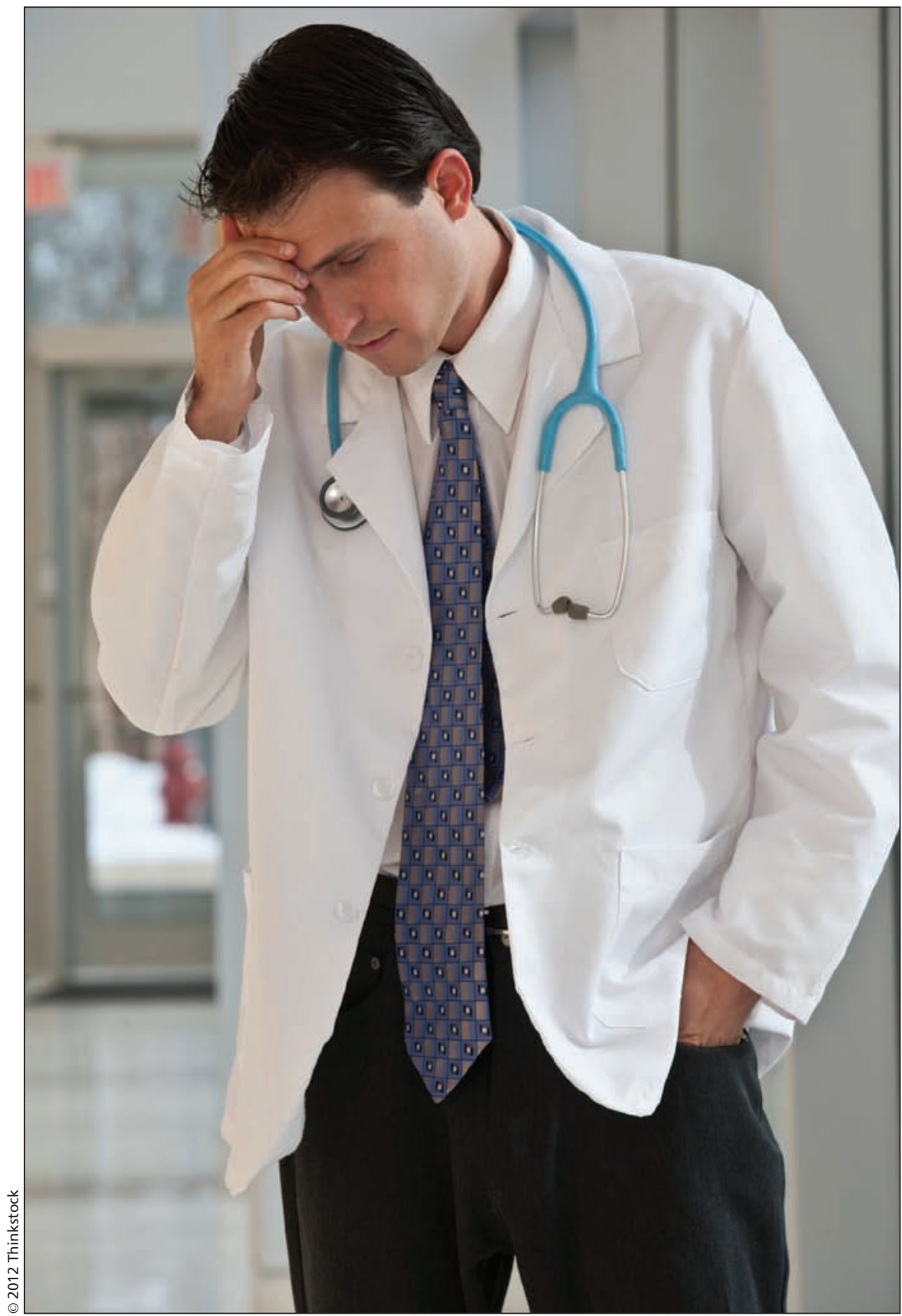

Many doctors in the US are abandoning private practice because they are fed up with administrative headaches, complex government regulations and nuanced payment methodologies.

ware company that provides electronic health records to small- and mediumsized hospitals across America.

But the reasons that private practice is vanishing are as numerous as the forms of large group practice, Esham adds. For some docs, "there is a sense of safety in being part of an organization," whether to shield themselves from economic uncertainty, share the burden of 
medical liability costs, afford the adoption of electronic medical records or even escape a nasty trend toward "profiling" physicians to determine who delivers the lowest cost care so as to funnel patients in their direction.

Also driving change is a US\$10billion innovation fund that was created as part of President Barack Obama's health care reforms to explore novel health delivery mechanisms, such as accountable care organizations (ACOs), as well as alternative means of payment for health professionals that might lower overall medical costs.

Another driver is that younger doctors, heavily burdened with medical school debt and wanting a better worklife balance, are turning to hospitals, integrated health networks, patient-centred medical homes and other forms of larger group practice for secure employment with defined hours of work.

Add to that the increasingly prohibitive cost of running a private practice, says Dr. M. Zuhdi Jasser, a primary care physician and chair of the AMA's private practice physicians caucus. "The free market aspect of medicine has disappeared," he says. "You have prices that are fixed and prices that are predetermined so the only institutions that can make any profit are the big ones that cost shift. If you're in a private practice, like I am, you get paid $\$ 40$ or $\$ 90$ or $\$ 150$ [per procedure]. That's it. My phone calls, my rent, my lab draws, my collections, my front desk, it's all unfunded."

"What's happening is we're slowly seeing the institutionalization of medicine and there are no incentives for small business," while many doctors can't afford the dwindling incomes and higher financial risks of private practice, so they're bailing to become employed docs, Jasser adds.

The AMA says less than one-third of American physicians will remain in private practice by 2013. The Medical Group Management Association says the number of medical practices that are physician-owned has dropped below $50 \%$. Because an increasing number of medical students are pursuing higherpaying specialties, many hospitals (sometimes networked with pharmaceutical firms and health insurance companies) are also becoming big buyers of physician practices and heavy recruiters of young physicians as they actively compete for an ever-diminishing pool of primary care doctors. The professional services giant PricewaterhouseCoopers says health insurance companies shelled out more than US $\$ 2$ billion in 2011 to purchase physician practices and projects the trend will only continue (www .pwc.com/en_US/us/transaction-services /publications/assets/pwc-ma-executive -agenda-february-2012.pdf).

According to the American Hospital Association, there was a $32 \%$ increase in the number of physicians employed by hospitals between 2000 and 2010 to about 212000 of America's nearly one million physicians, with the largest growth coming in the area of "hospitalists." In the three-year period ending in 2010, hospitals hired 10000 hospitalists, bolstering their inhouse ranks to 30000 .

A select group of physicians have been able to remain in private practice by opting to become "concierge or direct pay doctors" who refuse to treat patients paid by health insurance companies or government programs like Medicare and Medicaid (for the elderly and the poor, respectively), says Dr. Leah McCormack, a dermatologist in New York City who opted for just such a practice.

"There will always be people like me who just want to remain in private practice," she says. "But it's becoming difficult for most because the individual or small practice just can't comply with the regulations. And eventually they give up. And hospitals are buying up practices all over the place, all in preparation of getting massive amounts of money from the government to be an ACO, or a co-op or one of these other entities."

The trend toward employed doctors is expected to be further exacerbated in coming years with the rollout of new health delivery models such as the
ACOs, the first 27 of which were unveiled by the US government in March (www.cms.gov/apps/media/press /release. asp?Counter $=4333$ ). They're viewed as a potentially integral component of an overhaul of Medicare, under which direct payments to doctors will be reduced by $31 \%$ as of Jan. 1, 2013. ACOs will manage the health of regional populations of at least 5000 Medicare recipients for a minimum of three years and, in exchange, will receive incentive payments under what is called the "shared savings model," depending on their performance against quality and savings metrics. Simply put, if an ACO saves the government money by reducing unnecessary tests and procedures, it will get a cut of the savings, depending on how much risk it was willing to assume that it could achieve specified benchmarks. The US Department of Health and Human Services has estimated the scheme will reduce Medicare costs by US $\$ 960$ million over three years.

Enthusiasts call them the future face of American health care. But like other developments, they are drawing physicians away from private practice. Some 10000 doctors are part of the 27 pioneering ACOs. Equally notable is that just 13 of the 27 ACOs were physicianled, in part because setting up an ACO involved costs that only hospitals and other well-capitalized institutions could readily generate.

As to whether they'll yield improvements in health care or reduced costs, in comparison with a system based primarily on private practice, that appears entirely unknown.

"What you're seeing is a lot of experimentation," says Dr. Stephen Epstein, an emergency physician in Boston, Massachusetts. "But what's the most cost-effective model of providing the most appropriate health care? We don't know. And what we really haven't come to grips with is what are the true costs of this model, as compared to that model." - Wayne Kondro, CMAJ

CMAJ 2012. DOI:10.1503/cmaj.109-4235 